\title{
EQUIVALENCE OF STURM-LIOUVILLE PROBLEM WITH FINITELY MANY $\delta$-INTERACTIONS AND MATRIX EIGENVALUE PROBLEMS
}

\author{
ABDULLAH KABLAN*, MEHMET AKİF ÇETİN \\ Faculty of Arts and Sciences, Department of Mathematics, Gaziantep University, Gaziantep, 27310, Turkey \\ *Corresponding author: kablan@gantep.edu.tr

\begin{abstract}
The purpuse of this article is to show the matrix representations of Sturm-Liouville operators with finitely many $\delta$-interactions. We show that a Sturm-Liouville problem with finitely many $\delta$-interactions can be represented as a finite dimensional matrix eigenvalue problem which has the same eigenvalue with the former Sturm-Liouville operator. Moreover an example is also presented.
\end{abstract}

\section{INTRODUCTION}

Acording to classical spectral theory, a Sturm-Liouville problem (SLP) consisting of the equation

$$
-\left(p y^{\prime}\right)^{\prime}+q y=\lambda w y, \text { on } J=(a, b)
$$

and boundary conditions has infinite spectrum under some assumptions. Atkinson in his book [1] suggested that if the coefficients of SLP satisfy some conditions, the problem may have finite eigenvalues. Then in [2], Kong, Wu and Zettl obtained the following result: For every positive integer n, we can construct a class of regular self-adjoint and nonself-adjoint SLP with exactly $n$ eigenvalues by choosing $p$ and $w$ such that $1 / p$ and $w$ are alternatively zero on consecutive subintervals.

Recently, there has been much attention paid to the SLPs with finite spectrum. For a comprehensive treatment of the subject we refer the reader to the book by Zettl [3], and the papers by Kong, Wu and Zettl [2], Ao, Sun, and Zhang [4], [5] and Ao, Bo and Sun [6], [7]. In 2009, the equivalence of SLP with

Received $7^{\text {th }}$ September, 2017; accepted $20^{\text {th }}$ October, 2017; published $3^{\text {rd }}$ January, 2018.

2010 Mathematics Subject Classification. 34B24, 47A10, 15A18.

Key words and phrases. one-dimensional Schrödinger operator; finite spectrum; point interactions.

(C)2018 Authors retain the copyrights of their papers, and all open access articles are distributed under the terms of the Creative Commons Attribution License. 
a matrix eigenvalue problem was first constructed by Volkmer and Zettl in [8]. By equivalance of matrix eigenvalue problems for the SLPs with finite spectrum we mean to construct a matrix eigenvalue problem with exactly the same eigenvalues as the corresponding SLP. Then, the matrix representations of SLPs with finite spectrum are extended to various problems. For the SLPs see [8]- [11] and for fourth order boundary value problems see [12]- [16].

The goal of this paper is to find the matrix representation of the following Sturm-Liouville problem with finitely many $\delta$-interactions:

$$
-\left(p y^{\prime}\right)^{\prime}+\sum_{n=1}^{\infty} \alpha_{n} \delta\left(x-x_{n}\right) y+q y=\lambda w y, \text { on } \quad J=(a, b),
$$

where $J=\left(a, x_{1}\right) \cup\left(x_{1}, x_{2}\right) \cup \ldots \cup\left(x_{n}, b\right), x_{1}, \ldots, x_{n} \in(a, b)$ with $-\infty<a<b<\infty, \alpha_{j}$ 's are real numbers, $\delta(x)$ is the Dirac delta function and $\lambda \in \mathbb{C}$ is a spectral parameter. Sturm-Liouville equations with Dirac delta function potentials often appear in quantum mechanics. For example, such an equations had been used for modelling of atomic and molecular systems including atomic lattices, quantum heterostructures, semiconductors, organic fluorescent materials, solar cells etc. (see [17], [18], [19] and citations of them). Recently, we generalize the finite spectrum result to the problem (1.1) in [20]. The equation (1.1) is equivalent to the many-point boundary value problem, (see [19]). So we can understand problem (1.1) as studying the equation

$$
-\left(p y^{\prime}\right)^{\prime}+q y=\lambda w y, \text { on } J
$$

and $n$ transmission conditions

$$
C_{j} Y\left(x_{j}-\right)=Y\left(x_{j}+\right), \quad Y=\left[\begin{array}{c}
y \\
p y^{\prime}
\end{array}\right], \quad j=1,2, \ldots, n
$$

where $x_{j}$ 's are inner discontinuity points and

$$
C_{j}=\left[\begin{array}{cc}
1 & 0 \\
\alpha_{j} & 1
\end{array}\right]
$$

Additionally, let us consider the boundary conditions of the form

$$
A Y(a)+B Y(b)=0, \quad A, B \in M_{2}(\mathbb{C})
$$

where $A=\left(a_{i j}\right)_{2 \times 2}, B=\left(b_{i j}\right)_{2 \times 2}$ are complex valued $2 \times 2$ matrices and $M_{2}(\mathbb{C})$ denotes the set of square matrices of order 2 over $\mathbb{C}$. Here, the coefficients fulfill the following minimal conditions:

$$
r=\frac{1}{p}, q, w \in L(J, \mathbb{C})
$$

where $L(J, \mathbb{C})$ denotes the complex valued functions which are Lebesgue integrable on $J$. 
The BC (1.3) is said to be self-adjoint if the following two conditions are satisfied:

$$
\operatorname{rank}(A, B)=2, A E A^{*}=B E B^{*} \text { with } E=\left[\begin{array}{cc}
0 & -1 \\
1 & 0
\end{array}\right] .
$$

It is well known that under the condition (1.5), the BCs (1.3) fall into two disjoint classes: seperated and coupled. The seperated boundary conditions have the canonical representation:

$$
\begin{aligned}
& \cos \alpha y(a)-\sin \alpha\left(p y^{\prime}\right)(a)=0,0 \leq \alpha<\pi \\
& \cos \beta y(b)-\sin \beta\left(p y^{\prime}\right)(b)=0,0<\beta \leq \pi .
\end{aligned}
$$

The real coupled boundary conditions have the canonical representation:

$$
Y(b)=K Y(a) \text { with } K=\left(k_{s, t}\right)_{2 \times 2}, k_{s, t} \in \mathbb{R}, \operatorname{det}(K)=1 .
$$

Let $u=y$ and $v=\left(p y^{\prime}\right)$. Then we have the system representation of equation (1.2)

$$
u^{\prime}=r v, v^{\prime}=(q-\lambda w) u, \text { on } J
$$

\section{Matrix Representations of SLPs with Finitely Many $\delta$-Interactions}

Definition 2.1. A Sturm-Liouville equation with finitely many $\delta$-interactions (1.1) or equivalently the equation (1.2) with transmission condition (1.3) is said to be of Atkinson type if, for some integers $m_{j} \geq 1, j=$ $0,1, \ldots, n$, there exists a partition of the interval $J$

$$
\begin{aligned}
a= & x_{00}<x_{01}<x_{02}<\ldots<x_{0,2 m_{0}+1}=x_{1}, \\
x_{1}= & x_{10}<x_{11}<x_{12}<\ldots<x_{1,2 m_{1}+1}=x_{2}, \\
& \vdots \\
x_{n-1}= & x_{n-1,0}<x_{n-1,1}<x_{n-1,2}<\ldots<x_{n-1,2 m_{n-1}+1}=x_{n}, \\
x_{n}= & x_{n 0}<x_{n 1}<x_{n 2}<\ldots<x_{n, 2 m_{n}+1}=b
\end{aligned}
$$

such that for each $j \in\{0,1, \ldots, n\}$

$$
\begin{gathered}
r=\frac{1}{p}=0 \text { on }\left(x_{j, 2 k} ; x_{j, 2 k+1}\right], k=0,1, \ldots, m_{j}-1 \text { and }\left[x_{j, 2 m_{j}} ; x_{j, 2 m_{j}+1}\right), \\
\int_{x_{j, 2 k}}^{x_{j, 2 k+1}} w \neq 0, \int_{x_{j, 2 k}}^{x_{j, 2 k+1}} q \neq 0, k=0,1, \ldots, m_{j},
\end{gathered}
$$

and

$$
q=w=0 \text { on }\left[x_{j, 2 k+1} ; x_{j, 2 k+2}\right], \int_{x_{j, 2 k+1}}^{x_{j, 2 k+2}} r \neq 0, k=0,1, \ldots, m_{j}-1
$$


Our main aim in this section is to constract matrix eigenvalue problems in such a way that its eigenvalues are exactly the same as those of the corresponding SLPs with finitely many $\delta$-Interactions of Atkinson type.

Definition 2.2. A SLP with finitely many $\delta$-Interactions of Atkinson type is said to be equivalent to a matrix eigenvalue problem if the former has exactly the same eigenvalues as the latter.

We begin by stating some additional notation. For each $j \in\{0,1, \ldots, n\}$ given (2.1)-(2.3), let

$$
\begin{gathered}
p_{j k}=\left(\int_{x_{j, 2 k-1}}^{x_{j, 2 k}} r\right)^{-1}, k=1,2, \ldots, m_{j} ; \\
q_{j k}=\int_{x_{j, 2 k}}^{x_{j, 2 k+1}} q, w_{j k}=\int_{x_{j, 2 k}}^{x_{j, 2 k+1}} w, k=0,1, \ldots, m_{j} .
\end{gathered}
$$

and let introduce the notation

$$
m=\sum_{j=0}^{n} m_{j} .
$$

We note from (2.2) and (2.3) that $p_{j k}, w_{j k} \in \mathbb{R} \backslash\{0\}$, and no sign restrictions are imposed on them.

From (2.2) and (2.3) we can make the following observation: For any solution $u, v$ of $(1.9), u$ is constant on the intervals where $r$ is identically zero and $v$ is constant on the intervals where both $q$ and $w$ are both identically zero. Let

$$
\begin{aligned}
u_{0 k} & =u(x), x \in\left[x_{0,2 k} ; x_{0,2 k+1}\right], k=0,1, \ldots, m_{0}-1, \\
u_{0 m_{0}} & =u(x), x \in\left[x_{0,2 m_{0}} ; x_{0,2 m_{0}+1}\right), \\
u_{j 0} & =u(x), x \in\left(x_{j 0} ; x_{j 1}\right], j=1,2, \ldots, n \\
u_{j k} & =u(x), x \in\left[x_{j, 2 k} ; x_{j, 2 k+1}\right], k=1,2, \ldots, m_{j}-1, j=1,2, \ldots, n-1 \\
u_{j m_{j}} & =u(x), x \in\left[x_{j, 2 m_{j}} ; x_{j, 2 m_{j}+1}\right), j=0,1, \ldots, n-1 \\
u_{n k} & =u(x), x \in\left[x_{n, 2 k} ; x_{n, 2 k+1}\right], k=1,2, \ldots, m_{n} \\
v_{j k} & =v(x), x \in\left[x_{j, 2 k-1} ; x_{j, 2 k}\right), k=1,2, \ldots, m_{j}, j=0,1, \ldots, n
\end{aligned}
$$

and set

$$
v_{j 0}=v\left(x_{j 0}+\right), v_{j, m_{j}+1}=v\left(x_{j, 2 m_{j}+1}-\right), j=0,1, \ldots, n
$$

Lemma 2.1. Assume Eq. (1.2) is of Atkinson type. Then for each $j=0,1, \ldots, n$ and for any solution u, $v$ of Eq. (1.9), we have

$$
\begin{gathered}
p_{j k}\left(u_{j k}-u_{j, k-1}\right)=v_{j k}, k=1,2, \ldots, m_{j}, \\
v_{j, k+1}-v_{j k}=u_{j k}\left(q_{j k}-\lambda w_{j k}\right), k=0,1, \ldots, m_{j} .
\end{gathered}
$$


Conversely, for any solution $u_{j k}, k=0,1, \ldots, m_{j}$ and $v_{j k}, k=0,1, \ldots, m_{j}+1$ of system (2.8), (2.9), there is a unique solution $u(x)$ and $v(x)$ of Eq. (1.9) satisfying (2.6) and (2.7).

Proof. Relying on the first equation of (1.9), for $k=1,2, \ldots, m_{j}$, we have

$$
\begin{aligned}
u_{j k}-u_{j, k-1} & =u\left(x_{j, 2 k}\right)-u\left(x_{j, 2 k-2}\right)=\int_{x_{j, 2 k-2}}^{x_{j, 2 k}} u^{\prime}=\int_{x_{j, 2 k-2}}^{x_{j, 2 k}} r v \\
& =\int_{x_{j, 2 k-1}}^{x_{j, 2 k}} r v=v_{j k} \int_{x_{j, 2 k-1}}^{x_{j, 2 k}} r=v_{j k} / p_{j k} .
\end{aligned}
$$

This establishes (2.8). Similarly, from second equation of $(1.9)$, for $k=0,1, \ldots, m_{j}$, we have

$$
\begin{aligned}
v_{j, k+1}-v_{j k} & =v\left(x_{j, 2 k+1}\right)-v\left(x_{j, 2 k-1}\right)=\int_{x_{j, 2 k-1}}^{x_{j, 2 k+1}} v^{\prime}=\int_{x_{j, 2 k-1}}^{x_{j, 2 k+1}}(q-\lambda w) u \\
& =\int_{x_{j, 2 k}}^{x_{j, 2 k+1}}(q-\lambda w) u=u_{j k} \int_{x_{j, 2 k}}^{x_{j, 2 k+1}}(q-\lambda w)=u_{j k}\left(q_{j k}-\lambda w_{j k}\right),
\end{aligned}
$$

which gives (2.9).

On the other hand, if $u_{j k}, v_{j k}$ satisfy (2.8) and (2.9), then we define $u(x)$ and $v(x)$ according to (2.6) and (2.7), and then extend them continuously to the whole interval $J$ as a solution of (1.9) by integrating the equations in (1.9) over subintervals.

First, we consider SLP with transmission condition(1.2)-(1.4) with seperated BC (1.7).

Theorem 2.1. Assume $\alpha \in[0, \pi), \beta \in(0, \pi]$. Define an $(m+1) \times(m+1)$ tridiagonal block matrix

$$
P_{\alpha \beta}=\left[\begin{array}{cccccc}
M_{0} & & & & & \\
N_{1} & M_{1} & & & & \\
& N_{2} & M_{2} & & & \\
& & \ddots & \ddots & & \\
& & & N_{n} & M_{n} & \\
& & & & N_{n+1} & M_{n+1}
\end{array}\right]
$$

and diagonal matrices

$$
\begin{gathered}
Q_{\alpha \beta}=\operatorname{diag}\left(q_{00} \sin \alpha, q_{01}, \ldots, q_{0, m_{0}-1}, q_{0 m_{0}}+q_{10}, q_{11}, \ldots, q_{n, m_{n}-1}, q_{n m_{n}} \sin \beta\right), \\
W_{\alpha \beta}=\operatorname{diag}\left(w_{00} \sin \alpha, w_{01}, \ldots, w_{0, m_{0}-1}, w_{0 m_{0}}+w_{10}, w_{11}, \ldots, w_{n, m_{n}-1}, w_{n m_{n}} \sin \beta\right) .
\end{gathered}
$$

Then SLP with transmission conditions (1.2), (1.3), (1.7) is equivalent to matrix eigenvalue problem

$$
\left(P_{\alpha \beta}+Q_{\alpha \beta}\right) U=\lambda W_{\alpha \beta} U
$$


where $m$ is as defined in (2.5),

$$
U=\left[u_{00}, u_{01}, \ldots, u_{0 m_{0}}, u_{11}, \ldots, u_{1 m_{1}}, \ldots, u_{n 1}, \ldots, u_{n m_{n}}\right]^{T}
$$

and the matrices $M_{j}$ 's and $N_{j}$ 's are defined as follows:

$$
M_{0}=\left[p_{01} \sin \alpha+\cos \alpha, \quad-p_{01} \sin \alpha\right],
$$

for each $j=0,1, \ldots, n$ the $m_{j} \times 2$ matrices

$$
N_{j+1}=\left[\begin{array}{cc}
-p_{j 1} & p_{j 1}+p_{j 2} \\
0 & -p_{j 2} \\
0 & 0 \\
\vdots & \vdots \\
0 & 0
\end{array}\right],
$$

for each $j=0,1, \ldots, n-1$ the $m_{j} \times m_{j}$ matrices

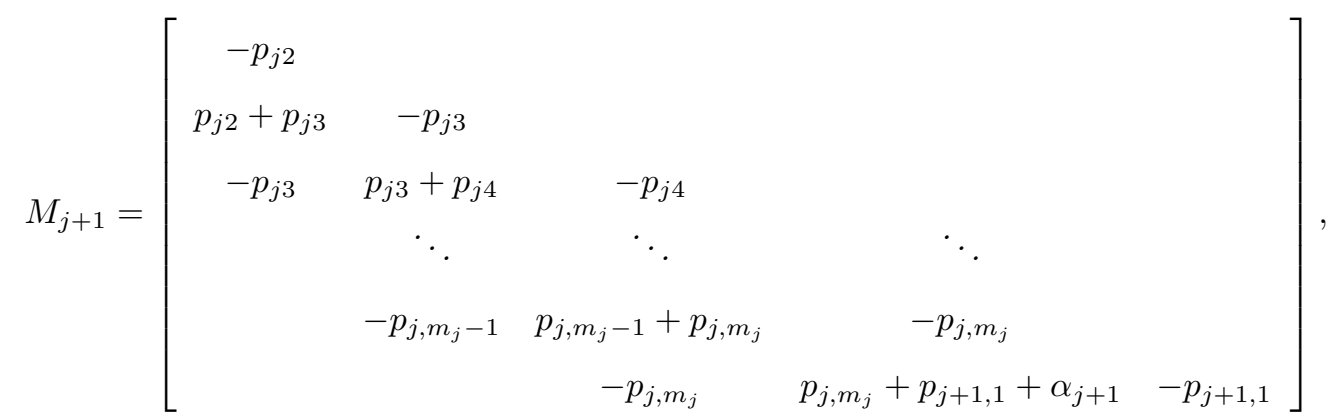

and the $m_{n} \times\left(m_{n}-1\right)$ matrix

$$
M_{n+1}=\left[\begin{array}{cccc}
-p_{n 2} & & & \\
p_{n 2}+p_{n 3} & -p_{n 3} & & \\
-p_{n 3} & p_{n 3}+p_{n 4} & -p_{n 4} & \\
& \ddots & \ddots & \ddots \\
& -p_{n, m_{n}-1} & p_{n, m_{n}-1}+p_{n, m_{n}} & -p_{n, m_{n}} \\
& & -p_{n, m_{n}} \sin \beta & p_{n, m_{n}} \sin \beta-\cos \beta
\end{array}\right] .
$$

Proof. For each $j=0,1, \ldots, n$ and $k=1,2, \ldots, m_{j}-1$, there is one-to-one correspondence between the solutions of system (2.8), (2.9) and the solutions of the following system:

$$
\begin{gathered}
p_{j 1}\left(u_{j 1}-u_{j 0}\right)-v_{j 0}=u_{j 0}\left(q_{j 0}-\lambda w_{j 0}\right), \\
p_{j, k+1}\left(u_{j, k+1}-u_{j k}\right)-p_{j k}\left(u_{j k}-u_{j, k-1}\right)=u_{j k}\left(q_{j k}-\lambda w_{j k}\right), \\
v_{j, m_{j}+1}-p_{j m_{j}}\left(u_{j m_{j}}-u_{j m_{j-1}}\right)=u_{j m_{j}}\left(q_{j m_{j}}-\lambda w_{j m_{j}}\right)
\end{gathered}
$$


Therefore, by Lemma 2.1, any solution of equation (1.9), and hence of (1.2), is uniquely determined by a solution of system (2.15)-(2.17). Note that from boundary condition (1.7), we have

$$
\begin{aligned}
u_{00} \cos \alpha & =v_{00} \sin \alpha \\
u_{n m_{n}} \cos \beta & =v_{n, m_{n}+1} \sin \beta
\end{aligned}
$$

and for each $j=0,1, \ldots, n-1$ from the transmission condition (1.3), we have

$$
\begin{aligned}
& u_{j+1,0}=u_{j m_{j}} \\
& v_{j+1,0}=\alpha_{j+1} u_{j m_{j}}+v_{j, m_{j}+1}
\end{aligned}
$$

Additionally, for each $j=0,1, \ldots, n-1$ from the equations (2.15)-(2.19), we have

$$
\begin{aligned}
& p_{j+1,1}\left(u_{j+1,1}-u_{j m_{j}}\right)-p_{j m_{j}}\left(u_{j m_{j}}-u_{j, m_{j}-1}\right)-u_{j m_{j}}\left(q_{j m_{j}}-\lambda w_{j m_{j}}\right) \\
&= \alpha_{j+1} u_{j m_{j}}+u_{j m_{j}}\left(q_{j+1,0}-\lambda w_{j+1,0}\right) \\
& p_{j+1,2}\left(u_{j+1,2}-u_{j+1,1}\right)-p_{j+1,1}\left(u_{j+1,1}-u_{j m_{j}}\right)=u_{j+1,1}\left(q_{j+1,1}-\lambda w_{j+1,1}\right) .
\end{aligned}
$$

Then the equivalence follows from (2.15)-(2.18) and (2.20), (2.21).

Corollary 2.1. Assume $\alpha, \beta \in(0, \pi)$. Define the $(m+1) \times(m+1)$ tridiagonal block matrix

$$
P_{\alpha \beta}=\left[\begin{array}{cccccc}
M_{0} & & & & & \\
N_{1} & M_{1} & & & & \\
& N_{2} & M_{2} & & & \\
& & \ddots & \ddots & & \\
& & & N_{n} & M_{n} & \\
& & & & N_{n+1} & M_{n+1}
\end{array}\right]
$$

and diagonal matrices

$$
\begin{gathered}
Q_{\alpha \beta}=\operatorname{diag}\left(q_{00}, q_{01}, \ldots, q_{0, m_{0}-1}, q_{0 m_{0}}+q_{10}, q_{11}, \ldots, q_{n, m_{n}-1}, q_{n m_{n}}\right) \\
W_{\alpha \beta}=\operatorname{diag}\left(w_{00}, w_{01}, \ldots, w_{0, m_{0}-1}, w_{0 m_{0}}+w_{10}, w_{11}, \ldots, w_{n, m_{n}-1}, w_{n m_{n}}\right) .
\end{gathered}
$$

Then SLP with transmission conditions (1.2), (1.3), (1.7) is equivalent to matrix eigenvalue problem

$$
\left(P_{\alpha \beta}+Q_{\alpha \beta}\right) U=\lambda W_{\alpha \beta} U
$$

where

$$
U=\left[u_{00}, u_{01}, \ldots, u_{0 m_{0}}, u_{11}, \ldots, u_{1 m_{1}}, \ldots, u_{n 1}, \ldots, u_{n m_{n}}\right]^{T}
$$


and the matrices $M_{j}$ 's and $N_{j}$ 's are defined as in Theorem 2.1 except $M_{0}$ and $M_{n+1}$. In this case, $M_{0}$ is $1 \times 2$ matrix

$$
M_{0}=\left[\begin{array}{ll}
p_{01}+\cot \alpha & -p_{01}
\end{array}\right]
$$

and $M_{n+1}$ is $m_{n} \times\left(m_{n}-1\right)$ matrix

$$
M_{n+1}=\left[\begin{array}{cccc}
-p_{n 2} & & & \\
p_{n 2}+p_{n 3} & -p_{n 3} & & \\
-p_{n 3} & p_{n 3}+p_{n 4} & -p_{n 4} & \\
& \ddots & \ddots & \ddots \\
& -p_{n, m_{n}-1} & p_{n, m_{n}-1}+p_{n, m_{n}} & -p_{n, m_{n}} \\
& & -p_{n, m_{n}} & p_{n, m_{n}}-\cot \beta
\end{array}\right] .
$$

Proof. If we divide the first and the last rows of system (2.10) by $\sin \alpha$ and $\sin \beta$ respectively, then we obtain $(2.22)$.

Theorem 2.1 and its Corollary show that the problem (1.2)-(1.4), (1.7) of Atkinson type have representations by tridiagonal matrix eigenvalue problems. Now, we will show that the problem (1.2)-(1.4), (1.8) of Atkinson type also have representations.

Theorem 2.2. Consider the boundary condition (1.8) with $k_{12}=0$. Define the $m \times m$ matrix which is tridiagonal except for the $(1, m)$ and $(m, 1)$ entries

$$
P_{1}=\left[\begin{array}{cccccc}
M_{0} & & & & & -k_{11} p_{n m_{n}} \\
N_{1} & M_{1} & & & & \\
& N_{2} & M_{2} & & & \\
& & \ddots & \ddots & & \\
& & & N_{n} & M_{n} & \\
& & & & N_{n+1} & M_{n+1}
\end{array}\right]
$$

and diagonal matrices

$$
\begin{gathered}
Q_{1}=\operatorname{diag}\left(q_{00}+k_{11}^{2} q_{n m_{n}}, q_{01}, \ldots, q_{0, m_{0}-1}, q_{0 m_{0}}+q_{10}, q_{11}, \ldots, q_{n m_{n}}\right), \\
W_{1}=\operatorname{diag}\left(w_{00}+k_{11}^{2} w_{n m_{n}}, w_{01}, \ldots, w_{0, m_{0}-1}, w_{0 m_{0}}+w_{10}, w_{11}, \ldots, w_{n m_{n}}\right) .
\end{gathered}
$$

Then SLP with transmission conditions (1.2), (1.3), (1.8) is equivalent to matrix eigenvalue problem

$$
\left(P_{1}+Q_{1}\right) U=\lambda W_{1} U
$$

where

$$
U=\left[u_{00}, u_{01}, \ldots, u_{0 m_{0}}, u_{11}, \ldots, u_{1 m_{1}}, \ldots, u_{n 1}, \ldots, u_{n, m_{n-1}}\right]^{T}
$$


and the elements of the matrix $P_{1}$ are defined as follows: The $1 \times 2$ matrix

$$
M_{0}=\left[\begin{array}{ll}
-k_{11} k_{21}+p_{01}+k_{11}^{2} p_{n m_{n}} & -p_{01}
\end{array}\right]
$$

for each $j=0,1, \ldots, n-1$ the $m_{j} \times 2$ and for $j=n$ the $\left(m_{n}-1\right) \times 2$ matrices

$$
N_{j+1}=\left[\begin{array}{cc}
-p_{j 1} & p_{j 1}+p_{j 2} \\
0 & -p_{j 2} \\
0 & 0 \\
\vdots & \vdots \\
0 & 0
\end{array}\right]
$$

for each $j=0,1, \ldots, n-1$ the $m_{j} \times m_{j}$ matrices

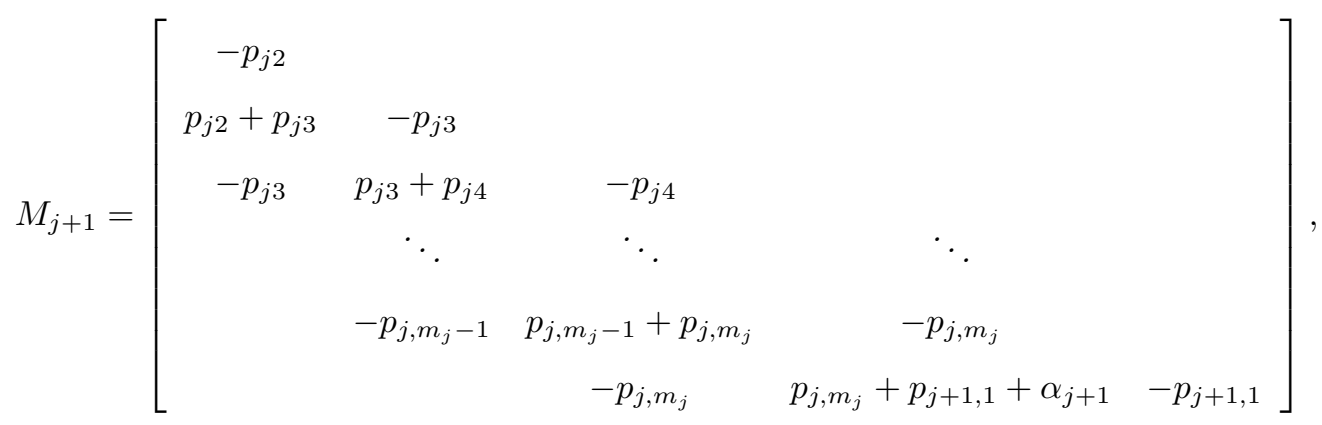

and the $\left(m_{n}-1\right) \times\left(m_{n}-2\right)$ matrix

$$
M_{n+1}=\left[\begin{array}{cccc}
-p_{n 2} & & & \\
p_{n 2}+p_{n 3} & -p_{n 3} & & \\
-p_{n 3} & p_{n 3}+p_{n 4} & -p_{n 4} & \\
& \ddots & \ddots & \ddots \\
& -p_{n, m_{n}-2} & p_{n, m_{n}-2}+p_{n, m_{n}-1} & -p_{n, m_{n}-1} \\
& & -p_{n, m_{n}-1} & p_{n, m_{n}-1}+p_{n m_{n}}
\end{array}\right]
$$

Proof. As mentioned before, the transmission condition (1.3) is the same as (2.19). On the other hand, since $k_{12}=0$, the boundary condition (1.8) is represented as follows:

$$
\begin{aligned}
u_{n m_{n}} & =k_{11} u_{00} \\
u_{n, m_{n}+1} & =k_{21} u_{00}+k_{22} v_{00}
\end{aligned}
$$

where $k_{11} k_{22}=1$. We find out that for each $j=0,1, \ldots, n-1$ and $k=0,1, \ldots, m_{j}-1$ there is one-to-one correspondence between the solutions consisting of system $(2.8),(2.9),(2.19),(2.24)$ and the solutions of the 
following system:

$$
\begin{gathered}
{\left[-k_{11} k_{21}+k_{11}^{2}\left(p_{j+1, m_{j+1}}+q_{j+1, m_{j+1}}-\lambda w_{j+1, m_{j+1}}\right)\right] u_{j 0}} \\
=\left(\lambda w_{j 0}-p_{j 1}-q_{j 0}\right) u_{j 0}+p_{j 1} u_{j 1}+k_{11} p_{j+1, m_{j+1}} u_{j+1, m_{j+1}-1} \\
p_{j, k+1}\left(u_{j, k+1}-u_{j k}\right)-p_{j k}\left(u_{j k}-u_{j, k-1}\right)=u_{j k}\left(q_{j k}-\lambda w_{j k}\right) \\
p_{j+1,1}\left(u_{j+1,1}-u_{j+1,0}\right)-v_{j+1,0}=u_{j+1,0}\left(q_{j+1,0}-\lambda w_{j+1,0}\right) \\
p_{j+1, m_{j+1}}\left(k_{11} u_{j 0}-u_{j+1, m_{j+1}-1}\right)-p_{j+1, m_{j+1}-1} u_{j+1, m_{j+1}-1} \\
=p_{j+1, m_{j+1}-1} u_{j+1, m_{j+1}-2}+u_{j+1, m_{j+1}-1}\left(q_{j+1, m_{j+1}-1}-\lambda w_{j+1, m_{j+1}-1}\right)
\end{gathered}
$$

Then, by Lemma 2.1, any solution of system (1.9), hence of (1.2), is uniquely determined by a solution of system (2.25)-(2.28).

Theorem 2.3. Consider the boundary condition (1.8) with $k_{12} \neq 0$. Define the $(m+1) \times(m+1) m a t r i x$ which is tridiagonal except for the $(1, m+1)$ and $(m+1,1)$ entries

$$
P_{2}=\left[\begin{array}{cccccc}
M_{0} & & & & & \frac{1}{k_{12}} \\
N_{1} & M_{1} & & & & \\
& N_{2} & M_{2} & & & \\
& & \ddots & \ddots & & \\
& & & N_{n} & M_{n} & \\
\frac{1}{k_{12}} & & & & N_{n+1} & M_{n+1}
\end{array}\right]
$$

and diagonal matrices

$$
\begin{gathered}
Q_{2}=\operatorname{diag}\left(q_{00}, q_{01}, \ldots, q_{0, m_{0}-1}, q_{0 m_{0}}+q_{10}, q_{11}, \ldots, q_{n, m_{n}-1}, q_{n m_{n}}\right) \\
W_{2}=\operatorname{diag}\left(w_{00}, w_{01}, \ldots, w_{0, m_{0}-1}, w_{0 m_{0}}+w_{10}, w_{11}, \ldots, w_{n, m_{n}-1}, w_{n m_{n}}\right)
\end{gathered}
$$

Then SLP with transmission conditions (1.2), (1.3), (1.8) is equivalent to matrix eigenvalue problem

$$
\left(P_{2}+Q_{2}\right) U=\lambda W_{2} U
$$

where

$$
U=\left[u_{00}, u_{01}, \ldots, u_{0 m_{0}}, u_{11}, \ldots, u_{1 m_{1}}, \ldots, u_{n 1}, \ldots, u_{n m_{n}}\right]^{T}
$$

and the elements of the matrix $P_{3}$ are defined as follows: For each $j=0,1, \ldots, n$ the matrices $N_{j+1}$ 's and for each $j=0,1, \ldots, n-1$ the matrices $M_{j+1}$ 's are defined as in Theorem 2.2. On the other hand, the $1 \times 2$ matrix

$$
M_{0}=\left[\begin{array}{ll}
p_{01}-\frac{k_{11}}{k_{12}} & -p_{01}
\end{array}\right]
$$


and the $m_{n} \times\left(m_{n}-1\right)$ matrix

$$
M_{n+1}=\left[\begin{array}{cccc}
-p_{n 2} & & & \\
p_{n 2}+p_{n 3} & -p_{n 3} & & \\
-p_{n 3} & p_{n 3}+p_{n 4} & -p_{n 4} & \\
& \ddots & \ddots & \ddots \\
& -p_{n, m_{n}-1} & p_{n, m_{n}-1}+p_{n m_{n}} & -p_{n m_{n}} \\
& & -p_{n m_{n}} & p_{n m_{n}}-\frac{k_{22}}{k_{12}}
\end{array}\right] .
$$

Proof. The boundary condition (1.8) can be represented as follows:

$$
\begin{aligned}
u_{n m_{n}} & =k_{11} u_{00}+k_{12} v_{00} \\
v_{n, m_{n}+1} & =k_{21} u_{00}+k_{22} v_{00} .
\end{aligned}
$$

Since $k_{11} k_{22}-k_{12} k_{21}=1$, we have from the this condition that

$$
\begin{aligned}
v_{00} & =-\frac{k_{11}}{k_{12}} u_{00}+\frac{1}{k_{12}} u_{n m_{n}}, \\
v_{n, m_{n}+1} & =-\frac{1}{k_{12}} u_{00}+\frac{k_{22}}{k_{12}} u_{n m_{n}} .
\end{aligned}
$$

On the other hand, if we consider the transmission condition (2.19), the proof is similar with Theorem 2.2 .

\section{ExAmple}

In this section, we give an example to illustrate that a SLP with finitely many $\delta$-interactions and it's equivalent matrix eigenvalue problem, we will construct it, have same eigenvalues.

Consider the SLP with $\delta$-interactions on $J=(-3,0) \cup(0,6)$,

$$
-\left(p y^{\prime}\right)^{\prime}+\delta(x-0) y+q y=\lambda w y
$$

This equation is equivalent to the following SLP

$$
-\left(p y^{\prime}\right)^{\prime}+q y=\lambda w y
$$

with transmission condition

$$
\left\{\begin{array}{c}
y(0-)-y(0+)=0 \\
y(0-)+p y^{\prime}(0-)-p y^{\prime}(0+)=0 .
\end{array}\right.
$$

By choosing $\alpha=0$ and $\beta=\pi$, we consider the following boundary conditions

$$
\left\{\begin{array}{c}
y(-3)=0 \\
y(6)=0
\end{array}\right.
$$


In this case, the matrices in (1.3) and (1.4) become

$$
C_{1}=\left[\begin{array}{ll}
1 & 0 \\
1 & 1
\end{array}\right], \quad A=\left[\begin{array}{ll}
1 & 0 \\
0 & 0
\end{array}\right], \quad B=\left[\begin{array}{ll}
0 & 0 \\
1 & 0
\end{array}\right]
$$

respectively. Now, let's take a partition of the interval $J$ as follows:

$$
\begin{aligned}
a & =-3<-2<-1<0=x_{1} \\
x_{1} & =0<2<3<4<5<6=x_{2}=b .
\end{aligned}
$$

This yields that $m_{0}=1, m_{1}=2$ and define the piecewise constant functions $p, q, w$ are as follows:

$$
p(x)=\left\{\begin{array}{cc}
\infty, & (-3,-2) \\
1, & (-2,-1) \\
\infty, & (-1,0) \\
\infty, & (0,2) \\
\frac{1}{2}, & (2,3) \\
\infty, & (3,4) \\
\frac{1}{4}, & (4,5) \\
\infty, & (5,6)
\end{array} \quad q(x)=\left\{\begin{array}{cc}
0, & (-3,-2) \\
0, & (-2,-1) \\
1, & (-1,0) \\
2, & (0,2) \\
0, & (2,3) \\
3, & (3,4) \\
0, & (4,5) \\
4, & (5,6)
\end{array} \quad w(x)= \begin{cases}1, & (-3,-2) \\
0, & (-2,-1) \\
3, & (-1,0) \\
4, & (0,2) \\
0, & (2,3) \\
1, & (3,4) \\
0, & (4,5) \\
2, & (5,6)\end{cases}\right.\right.
$$

By using the similar method as given in [4], [5] or [20] we have the following two eigenvalues

$$
\lambda_{1}=0.67442, \quad \lambda_{2}=3.75739
$$

On the other hand, if we find the values $p_{j k}, q_{j k}, w_{j k}$ from (2.4), and use Theorem 2.1 we get the matrices

$$
P_{0 \pi}=\left[\begin{array}{cccc}
1 & 0 & 0 & 0 \\
-1 & \frac{5}{2} & -\frac{1}{2} & 0 \\
0 & -\frac{1}{2} & \frac{3}{4} & -\frac{1}{4} \\
0 & 0 & 0 & -1
\end{array}\right], Q_{0 \pi}=\left[\begin{array}{cccc}
0 & 0 & 0 & 0 \\
0 & 5 & 0 & 0 \\
0 & 0 & 3 & 0 \\
0 & 0 & 0 & 0
\end{array}\right], W_{0 \pi}=\left[\begin{array}{cccc}
0 & 0 & 0 & 0 \\
0 & 11 & 0 & 0 \\
0 & 0 & 1 & 0 \\
0 & 0 & 0 & 0
\end{array}\right],
$$

and so the matrix eigenvalue problem

$$
\left(P_{0 \pi}+Q_{0 \pi}\right) U=\lambda W_{0 \pi} U
$$

which is equivalance of SLP with finitely many $\delta$-interactions in (3.1). Indeed, if we find the eigenvalues of the matrix eigenvalue problem (3.9) we obtain the eigenvalues in (3.7). 


\section{REFERENCES}

[1] F.V. Atkinson, Discrete and Continuous Boundary Problems, Academic Press-New York, London, 1964.

[2] Q. Kong, H. Wu and A. Zettl, Sturm-Liouville Problems with Finite Spectrum, J. Math. Anal. Appl. 263 (2001), 748-762.

[3] A. Zettl, Sturm-Liouville Theory, Amer. Math. Soc., Math. Surveys Monographs, no. 121, 2005.

[4] J.J. Ao, J. Sun and M.Z. Zhang, The Finite Spectrum of Sturm-Liouville Problems with Transmission Conditions, Appl. Math. Comput. 218 (2011), 1166-1173.

[5] J.J. Ao, J. Sun and M.Z. Zhang, The Finite Spectrum of Sturm-Liouville Problems with Transmission Conditions and Eigenparameter-Dependent Boundary Conditions, Result. Math. 63(3-4) (2013), 1057-1070.

[6] J.J. Ao, F.Z. Bo and J. Sun, Fourth-Order Boundary Value Problems with Finite Spectrum, Appl. Math. Comput. 244 (2014), 952-958.

[7] F.Z. Bo and J.J. Ao, The Finite Spectrum of Fourth-Order Boundary Value Problems with Transmission Conditions, Abstr. Appl. Anal. 2014 (2014), Art. ID 175489.

[8] Q. Kong, H. Volkmer and A. Zettl, Matrix Representations of Sturm-Liouville Problems with Finite Spectrum, Result. Math. 54 (2009), 103-116.

[9] J.J. Ao, J. Sun and M.Z. Zhang, Matrix Representations of Sturm-Liouville Problems with Transmission Conditions, Comput. Math. Appl. 63 (2012), 1335-1348.

[10] J.J. Ao. and J. Sun, Matrix Representations of Sturm-Liouville Problems with Eigenparameter-Dependent Boundary Conditions, Linear Algebra Appl. 438 (2013), 2359-2365.

[11] J.J. Ao. and J. Sun, Matrix Representations of Sturm-Liouville Problems with Coupled Eigenparameter-Dependent Boundary Conditions, Appl. Math. Comput. 244 (2014), 142-148.

[12] J.J. Ao, J. Sun and A. Zettl, Matrix Representations of Fourth-Order Boundary Value Problems with Finite Spectrum, Linear Algebra Appl. 436 (2012), 2359-2365.

[13] J.J. Ao, J. Sun and A. Zettl, Equivalance of Fourth-Order Boundary Value Problems and Matrix Eigenvalue Problems, Result. Math. 63 (2013), 581-595.

[14] S. Ge, W. Wang and J.J. Ao, Matrix Representations of Fourth-Order Boundary Value Problems with Periodic Boundary Conditions, Appl. Math. Comput. 227 (2014), 601-609.

[15] A. Kablan and M. D. Manafov, Matrix Representations of Fourth-Order Boundary Value Problems with Transmission Conditions, Mediterranean J. Math. 13(1) (2016), 205-215.

[16] J.J. Ao. and J. Sun, Matrix Representations of Fourth-Order Boundary Value Problems with Coupled or Mixed Boundary Conditions, Linear Multilinear Algebra 63(8) (2015), 1590-1598.

[17] L.N. Pandey and T.F. George, Intersubband Transitions in Quantum Well Heterostructures with Delta-Doped Barriers, Appl. Phys. Lett. 61 (2016), 1081.

[18] R.K. Willardson and A.C. Beer, Semiconductors and Semimetals, Academic Press, London, 1984.

[19] S. Albeverio, F. Gesztesy, R. Høegh-Krohn and H. Holden, Solvable Models in Quantum Mechanics, Springer-Verlag, Berlin/New York, 1988.

[20] A. Kablan, M.A. Çetin and M.D. Manafov, The Finite Spectrum of Sturm-Liouville Operator with Finitely Many $\delta$ interactions, pre-print. 